\title{
$q$-Deformed Heisenberg-Algebra
}

\author{
Julius Wess \\ Sektion Physik der Ludwig-Maximilians-Universität \\ Theresienstr. 37, D-80333 München \\ and \\ Max-Planck-Institut für Physik \\ (Werner-Heisenberg-Institut) \\ Föhringer Ring 6, D-80805 München
}

ABSTRACT: Quantum group considerations lead to a discrete spacetime structure. This structure can be imbedded into $\mathbf{R}^{n}$. The simplest model that shows these features is based on the one dimensional $q$-deformed Heisenberg algebra and will be discussed in this lecture.

The canonical commutation relations are at the basis of a quantum mechanical system:

$$
[\hat{x}, \hat{p}]=\hat{x} \hat{p}-\hat{p} \hat{x}=i
$$

The elements of this algebra are supposed to be selfadjoint

$$
\overline{\hat{x}}=\hat{x} \quad, \quad \overline{\hat{p}}=\hat{p}
$$

A physical system is defined through a representation of this algebra in a Hilbertspace where selfadjoint elements of the algebra have to be represented by (essentially) selfadjoint linear operators.

In quantum mechanics the elements of (1, $\left.1 \overline{1}_{1}^{\prime}\right)$ are represented in the Hilbertspace of squareintegrable functions by:

$$
\hat{x}=x \quad, \quad \hat{p}=-i \frac{\partial}{\partial x}
$$

Starting from the algebra $\left(\begin{array}{l}\overline{1} \\ 1\end{array}\right)$, the spectrum of the linear operator $\hat{x}$ can be interpreted as the manifold on which the physical system lives - i.e. the configuration space.

In quantum mechanics it is $\mathbb{R}_{1}\left(x \in \mathbb{R}_{1}\right)$. The element $\hat{p}$ is a differential operator on this manifold.

We shall change the algebra ('i $\left.\overline{1}_{1}^{\prime}\right)$ in accord with quantum group considerations. It is natural to assume that $\hat{x}$ is an element of a quantum plane and to relate $\hat{p}$ to a derivative in such a plane. With an obvious change in notation we study the algebra

$$
\partial x=1+q x \partial
$$

More precisely, we study the free algebra generated by the elements $x$ and $\partial$ and divided by the

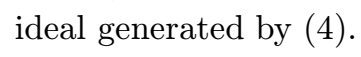

If we assume $x$ to be selfadjoint

$$
\bar{x}=x
$$

we see that this cannot be the case for $i \partial$ because

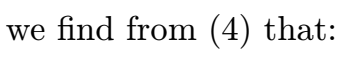

$$
\bar{\partial} x=-\frac{1}{q}+\frac{1}{q} x \bar{\partial}
$$

Thus we could study the algebra generated by $x, \partial$ and $\bar{\partial}$ and divide by $\left(\overline{\alpha_{1}^{i}}\right),\left(\overline{6}_{1}^{\bar{n}}\right)$ and an ideal generated by $\partial \bar{\partial}$ relations. We compute from ( $\left(\bar{A} \mathbf{A}_{1}\right)$ and $\left(\bar{b}^{-1}\right) \partial \bar{\partial} x$ and $\bar{\partial} \partial x$ and find that

$$
\bar{\partial} \partial=q \partial \bar{\partial}
$$

is consistent with these calculations. If we now try to define an operator $\hat{p}$ by $\hat{p}=-\frac{i}{2}(\partial-\bar{\partial})$ we find that the $x, \hat{p}$ relations do not close. The real part of $\partial$ has to be introduced as well. Thus our Heisenberg algebra would have one space and two momentum operators - a system that will hardly find a physical interpretation.

It turns out that $\bar{\partial}$ can be related to $\partial$ and $x$ in a nonlinear way. This relation involves the 
scaling operator $\Lambda$ :

$$
\begin{aligned}
\Lambda & \equiv q^{\frac{1}{2}}(1+(q-1) x \partial) \\
\Lambda x & =q x \Lambda \\
\Lambda \partial & =q^{-1} \partial \Lambda
\end{aligned}
$$

The scaling property follows from $\left(\begin{array}{l}\overline{4} \\ -1\end{array}\right)$.

We now define

$$
\tilde{\partial}=-q^{-\frac{1}{2}} \Lambda^{-1} \partial
$$

$\Lambda^{-1}$ is defined by an expansion in $(q-1)$. We find

$$
\begin{aligned}
& \tilde{\partial} x=-\frac{1}{q}+\frac{1}{q} x \tilde{\partial} \\
& \tilde{\partial} \partial=q \partial \tilde{\partial}
\end{aligned}
$$

Comparing this with $\left(\overline{\overline{6}_{1}}\right)$ ) and $\left(\overline{\overline{7}_{1}}\right)$ it follows from $\left(\bar{g}_{1}^{\prime}\right)$ and $\left(\overline{1} \overline{0}_{1}^{\prime}\right)$ that conjugation in the $x, \partial$ algebra can be defined by

$$
\bar{x}=x \quad, \quad \bar{\partial}=-q^{-\frac{1}{2}} \Lambda^{-1} \partial
$$

Conjugating $\Lambda$ and using $\left({ }_{1}^{1} \overline{1}_{1}\right)$ shows that

$$
\bar{\Lambda}=\Lambda^{-1}
$$

$\Lambda$ is a unitary element of the algebra, this justifies the factor $q^{\frac{1}{2}}$ in the definition of $\Lambda$.

The existence of a scaling operator $\Lambda$ and the definition of the conjugation $\left(\overline{1}_{1} \overline{1}_{1}^{1}\right)$ seems to be very specific for the $x, \partial$ algebra $\left(\begin{array}{l}(14 \\ 1\end{array}\right)$. It is however generic in the sense that a scaling operator and a definition of conjugation based on it can be found for all the quantum planes defined by $S O_{q}(n)$ and $S O_{q}(1, n)$.

The definition of the $q$-deformed Heisenberg algebra will now be based on the definition of the momentum:

$$
p=-\frac{i}{2}(\partial-\bar{\partial})
$$

It is selfadjoint. From the $x, \partial$ algebra and the definition of $\bar{\partial}$ follows

$$
\begin{aligned}
& q^{\frac{1}{2}} x p-q^{-\frac{1}{2}} p x=i \Lambda^{-1} \\
& \Lambda x=q x \Lambda \quad \Lambda p=q^{-1} p \Lambda
\end{aligned}
$$

and

$$
\bar{p}=p \quad, \quad \bar{x}=x \quad, \quad \bar{\Lambda}=\Lambda^{-1}
$$

These algebraic relations can be verified in the $x, \partial$ representation where the ordered $x, \partial$ monomials form a basis. We shall take (11 $\left.\overline{4}_{1}^{\prime}\right)$ and
(15) as the defining relations for the $q$-deformed Heisenbergalgebra without making further reference to its $x, \partial$ representation.

Let us discuss the representations of (114i). We assume $p$ to be represented by an essentially selfadjoint operator and therefore diagonalyzable. We assume $p$ to be diagonal. Let $p_{0}$ be an eigenvalue and $|0\rangle$ its eigenstate.

$$
p|0\rangle=p_{0}|0\rangle
$$

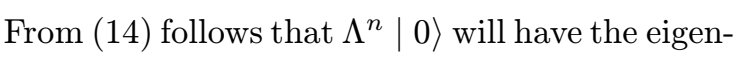
value $q^{n} p_{0}$

$$
\begin{aligned}
p|n\rangle & =q^{n} p_{0}|n\rangle \\
\Lambda|n\rangle & =|n+1\rangle
\end{aligned}
$$

As $\Lambda$ is unitary and $p$ selfadjoint we find:

$$
\langle m \mid n\rangle=\delta_{m n}
$$

As Hilbertspace $H_{p_{0}}$ we take the completion of the linear span $D_{p_{0}}$

$$
\begin{gathered}
D_{p_{0}}=\left\{\sum_{\text {finite }} c_{n}|n\rangle\right\} \\
H_{p_{0}}=\left\{\sum_{n=-\infty}^{\infty} c_{n}|n\rangle, \sum_{n=-\infty}^{\infty}\left|c_{n}\right|^{2}<\infty\right\}
\end{gathered}
$$

On this Hilbertspace $x$ can be represented as follows:

$$
\begin{aligned}
& x|n\rangle \\
& =\frac{i}{p_{0}} \frac{q^{-n}}{q-\frac{1}{q}}\left(q^{\frac{1}{2}}|n-1\rangle-q^{-\frac{1}{2}}|n+1\rangle\right)
\end{aligned}
$$

The eqns $\left(\overline{1}_{1}^{1} \overline{\vec{z}}_{1}\right)$ and $\left(\overline{2}_{2} \overline{0}_{1}^{\prime}\right)$ define a representation of the algebra (14). In this representation $p$ is essentially selfadjoint and $\Lambda$ is unitary. The operator $x$ is symmetric:

$$
\overline{\langle m|x| n\rangle}=\langle n|x| m\rangle
$$

It follows from (1] $\left.{ }_{2}^{1} \bar{A}_{i}\right)$ that another representation of the algebra can be otained by defining

$$
p^{s}= \pm s p \quad, \quad x^{s}= \pm s^{-1} x \quad, \quad \Lambda^{s}=\Lambda
$$

where $s$ is a real number. For $1 \leq s<q$ (we now consider the case $q \geq 1$ ) the representations obtained that way will be inequivalent. 
We now show that $x$ cannot be essentially selfadjoint in these representations. We assume that it is and thus can be diagonalized. Let | $\left.x_{0}\right\rangle$ be an eigenvector with eigenvalue $x_{0}$. The operator $\Lambda$ lowers and $\Lambda^{-1}$ raises this eigenvalue. The states obtained that way are orthogonal $(x$ essentially selfadjoint).

$$
\begin{gathered}
x\left|x_{0}\right\rangle=x_{0}\left|x_{0}\right\rangle \\
\left|x_{0}\right\rangle=\sum_{n=-\infty}^{\infty} c_{n}|n\rangle, \sum_{n=-\infty}^{\infty}\left|c_{n}\right|^{2}=1
\end{gathered}
$$

From the algebra follows

$$
\begin{gathered}
\left\langle x_{0}|p| x_{0}\right\rangle x_{0}\left(q^{\frac{1}{2}}-q^{-\frac{1}{2}}\right)=0 \\
s p_{0} \sum_{n=-\infty}^{\infty} q^{n}\left|c_{n}\right|^{2}=0
\end{gathered}
$$

This contradicts ( $(2 \overline{3})$.

The story is that $x$ is symmetric but not essentially selfadjoint. It can be shown that $x$ has exactly one eigenvector for each of the eigenvalues $\pm i$. Thus it has a one-parameter family of selfadjoint extensions. But none of these extensions will represent the algebra. This also follows from the argument given above. It is however possible to find reducible representations of the algebra (14) where the selfadjoint extension of $x$ will satisfy the algebra (114i). It is obvious from (24) that this is only possible if $p_{0}$ is allowed to have both signs. This will be discussed in the next section.

We now show that the algebra (i 1 (i) be represented in the "quantum mechanical" Hilbertspace defined by $(\underline{1} \overline{1}(\overline{1})$. With the notation

$$
\hat{z}=-\frac{i}{2}(\hat{p} \hat{x}+\hat{x} \hat{p})=-i \hat{p} \hat{x}+\frac{1}{2}=-i \hat{x} \hat{p}-\frac{1}{2}
$$

and, for any quantity $A$ :

$$
[A]=\frac{q^{A}-q^{-A}}{q-q^{-1}}
$$

we define

$$
\begin{gathered}
p=\hat{p} \\
x=\frac{\left[\hat{z}+\frac{1}{2}\right]}{\left(\hat{z}+\frac{1}{2}\right)} \hat{x} \\
\Lambda=q^{-\hat{z}}
\end{gathered}
$$

and show that $p, x$ satisfy the algebraic relations (114is) if $\hat{p}$ and $\hat{x}$ satisfy $\left(\overline{1}_{1}^{\prime}\right)$. We start from:

$$
\hat{x} \hat{z}=(\hat{z}+1) \hat{x} \quad, \quad \hat{p} \hat{z}=(\hat{z}-1) \hat{p}
$$

and conclude:

$$
\hat{x} f(\hat{z})=f(\hat{z}+1) \hat{x} \quad, \quad \hat{p} f(\hat{z})=f(\hat{z}-1) \hat{p}
$$

Let us now verify the first relation of $\left(\overline{1}_{1}^{\prime}\right)$.

$$
\begin{gathered}
q^{\frac{1}{2}} \frac{\left[\hat{z}+\frac{1}{2}\right]}{\left(\hat{z}+\frac{1}{2}\right)} \hat{x} \hat{p}-q^{-\frac{1}{2}} \hat{p} \frac{\left[\hat{z}+\frac{1}{2}\right]}{\left(\hat{z}+\frac{1}{2}\right)} \hat{x}= \\
q^{\frac{1}{2}} \frac{\left[\hat{z}+\frac{1}{2}\right]}{\left(\hat{z}+\frac{1}{2}\right)} \hat{x} \hat{p}-q^{-\frac{1}{2}} \frac{\left[\hat{z}-\frac{1}{2}\right]}{\left(\hat{z}-\frac{1}{2}\right)} \hat{p} \hat{x} \\
=i q^{\frac{1}{2}}\left[\hat{z}+\frac{1}{2}\right]-q^{-\frac{1}{2}}\left[\hat{z}-\frac{1}{2}\right]=i q^{\hat{z}}=i \Lambda^{-1}
\end{gathered}
$$

The other relations are verified in the same way. That $p$ is selfadjoint and $\Lambda$ unitary if $\hat{p}$ and $\hat{x}$ are selfadjoint is obvios. Let us show that $x$ is selfadjoint as well:

$$
\begin{aligned}
\hat{x}=\hat{x} \frac{\left[-\hat{z}+\frac{1}{2}\right]}{\left(-\hat{z}+\frac{1}{2}\right)} & =\hat{x} \frac{\left[\hat{z}-\frac{1}{2}\right]}{\left(\hat{z}-\frac{1}{2}\right)} \\
& =\frac{\left[\hat{z}+\frac{1}{2}\right]}{\left(\hat{z}+\frac{1}{2}\right)} \hat{x}=x
\end{aligned}
$$

The $x, p, \Lambda$ algebra does not change if $\hat{x} \hat{p}$ are changed by a canonical transformation. A class of such canonical transformations is:

$$
\tilde{p}=f(\hat{z}) \hat{p} \quad, \quad \tilde{x}=\tilde{x} f^{-1}(\hat{x})
$$

We immediately see that

$$
\tilde{x} \tilde{p}-\tilde{p} \tilde{x}=i
$$

The elements $\tilde{p}, \tilde{x}$ will be selfadjoint if

$$
\bar{f}(\overline{\hat{z}})=f(\hat{z}+1)
$$

An example of such an $f$ is:

$$
f^{-1}(\hat{z})=\frac{\left[\hat{z}-\frac{1}{2}\right]}{\hat{z}-\frac{1}{2}}
$$

With such a canonical transformation we find:

$$
\begin{gathered}
p=\frac{\left[\tilde{z}-\frac{1}{2}\right]}{\left(\tilde{z}-\frac{1}{2}\right)} \tilde{p} \\
x=\tilde{x} \\
\Lambda=q^{-\tilde{z}}
\end{gathered}
$$


This is again a representation of the $x p \Lambda$ algebra.

Let us see how the representation $\left(\overline{2} \overline{7}_{1}^{\prime}\right)$ is related to the representations $\left(\overline{1}_{1} \overline{7}_{1}^{\prime}\right),\left(\overline{2} \overline{0}_{1}^{\prime}\right),\left(\overline{2} \overline{2}_{1}^{\prime}\right)$. We start from a representation of (1-1) where $\hat{p}$ is diagonal:

$$
\hat{p}\left|k_{0}\right\rangle=k_{0}\left|k_{0}\right\rangle \quad\left\langle k_{0}^{\prime} \mid k_{0}\right\rangle=\delta\left(k^{\prime}-k_{0}\right)
$$

Eqn. $\left(\overline{2} \overline{2}_{1}^{i}\right)$ suggests the notation:

$$
\begin{aligned}
& p^{s}|n, \sigma\rangle|s\rangle=\sigma s q^{n} p_{0}|n, \sigma\rangle|s\rangle \\
& x^{s}|n, \sigma\rangle|s\rangle=\sigma s^{-1} p_{0}^{-1} q^{-n} \frac{i}{q-\frac{1}{q}} \\
&\left(q^{\frac{1}{2}}|n-1, \sigma\rangle|s\rangle-q^{-\frac{1}{2}}|n+1, \sigma\rangle|s\rangle\right) \\
&\left\langle s \mid s^{\prime}\right\rangle=\delta\left(s-s^{\prime}\right) \\
& \sigma= \pm 1 \quad, \quad 1 \leq s<q .
\end{aligned}
$$

The representation $\left(\overline{2}_{\mathbf{2}} \overline{\overline{7}_{1}}\right)$ can be reduced:

$$
|n, \sigma\rangle|s\rangle=\int d k_{0} q^{\frac{n}{2}} \delta\left(k_{0}-\sigma s q^{n}\right)\left|k_{0}\right\rangle
$$

Normalization:

$$
\begin{aligned}
& \left\langle n^{\prime}, \sigma^{\prime}\left|\left\langle s^{\prime} \| n, \sigma\right\rangle\right| s\right\rangle \\
& =\int d k_{0} d k_{0}^{\prime} q^{\frac{1}{2}\left(n+n^{\prime}\right)} \\
& \quad \delta\left(k_{0}-k_{0}^{\prime}\right) \delta\left(k_{0}-\sigma s q^{n}\right) \delta\left(k_{0}^{\prime}-\sigma^{\prime} s^{\prime} q^{n^{\prime}}\right) \\
& =\int d k_{0} q^{\frac{1}{2}\left(n+n^{\prime}\right)} \delta\left(k_{0}-\sigma s q^{n}\right) \delta\left(k_{0}-\sigma^{\prime} s^{\prime} q^{n^{\prime}}\right) \\
& =q^{\frac{1}{2}\left(n+n^{\prime}\right)} \delta\left(\sigma^{\prime} s^{\prime} q^{n^{\prime}}-\sigma s q^{n}\right) \\
& =q^{\frac{1}{2}\left(n+n^{\prime}\right)} \frac{1}{q^{n}} \delta_{n, n^{\prime}} \delta_{\sigma, \sigma^{\prime}} \delta\left(s-s^{\prime}\right) \\
& =\delta_{n, n^{\prime}} \delta_{\sigma, \sigma^{\prime}} \delta\left(s-s^{\prime}\right) .
\end{aligned}
$$

The transformation ( $\left.\bar{B}^{3} \overline{8}_{-}^{\prime}\right)$ can be inverted:

$$
\begin{aligned}
& \left|k_{0}\right\rangle= \\
& \int_{1}^{s} d s \sum_{n=-\infty}^{\infty} \sum_{\sigma=+,-} q^{\frac{n}{2}} \delta\left(k_{0}-\sigma s q^{n}\right)|n, \sigma\rangle|s\rangle
\end{aligned}
$$

Let us now act with $\Lambda$ as defined by $\left(12 \overline{7}_{1}\right)$ on $\left(\bar{B}_{2}^{\prime \prime}\right)$. On a wavefunction in the $k_{0}$ representation (36 $\left.{ }^{i}\right)$ $\hat{x}$ acts as $i \frac{\partial}{\partial k_{0}}$. The action of $\Lambda$ then is

$$
\begin{aligned}
\Lambda f\left(k_{0}\right) & =q^{-\frac{1}{2}} q^{-k_{0} \frac{\partial}{\partial k_{0}}} f\left(k_{0}\right) \\
& =q^{-\frac{1}{2}} f\left(q^{-1} k_{0}\right) \\
\Lambda q^{\frac{n}{2}} \delta\left(k_{0}-\sigma s q^{n}\right) & =q^{-\frac{1}{2}} q^{\frac{n}{2}} \delta\left(q^{-1} k_{0}-\sigma s q^{n}\right) \\
& =q^{\frac{1}{2}(n+1)} \delta\left(k_{0}-\sigma s q^{(n+1)}\right)
\end{aligned}
$$$$
\Lambda|n \sigma\rangle|s\rangle=|n+1, \sigma\rangle|s\rangle
$$

The action of $x$ can be otained from $\left(\overline{4} \overline{1}_{1}^{\prime}\right)$ by

$$
x=\frac{i}{q-\frac{1}{q}}\left(q^{\frac{1}{2}} \Lambda^{-1}-q^{-\frac{1}{2}} \Lambda\right) p^{-1}
$$

Its action on $|n, \sigma\rangle|s\rangle$ is identical with (3. $\left.\bar{z}_{1}\right)$. This shows that the representation (2 $\left.\overline{2}_{1}\right)$ decomposes into a direct integral of representations of the type $\left(\underline{2}_{2}^{2} \bar{O}_{i}^{i}\right)$ and $(\overline{2} \overline{2} \overline{2} i)$. 